\title{
Modeling fibrous cap formation in atherosclerotic plaque development: stability and oscillatory behavior
}

\author{
Wanwarat Anlamlert ${ }^{1}$, Yongwimon Lenbury ${ }^{2,3^{*}}$ and Jonathan Bell ${ }^{4}$
}

"Correspondence:
yongwimon.len@mahidol.ac.th
${ }^{2}$ Centre of Excellence in
Mathematics, PERDO, CHE, Bangkok,
Thailand
${ }^{3}$ Department of Mathematics,
Faculty of Science, Mahidol
University, Rama 6 Road, Bangkok,
Thailand
Full list of author information is
available at the end of the article

\section{Springer}

\begin{abstract}
Atherosclerosis usually occurs within the large arteries. It is characterized by the inflammation of the intima, which involves dynamic interactions between the plasma molecules; namely, LDL (low density lipoproteins), monocytes or macrophages, cellular components and the extracellular matrix of the arterial wall. This process is referred to as plaque formation. If the accumulation of $L D L$ cholesterol progresses unchecked, atherosclerotic plaques will form as a result of increased number of proliferating smooth muscle cells (SMCs) and extracellular lipid. This can thicken the artery wall and interfere further with blood flow. The growth of the plaques can become thrombotic and unstable, ending in rupture which gives rise to many life threatening illnesses, such as coronary heart disease, cardiovascular diseases, myocardial infarction, and stroke. A mathematical model of the essential chemical processes associated with atherosclerotic plaque development is analyzed, considering the concentrations of LDLs, oxidized LDLs, foam cells, oxidized LDL-derived chemoattractant and macrophage-derived chemoattractant, the density of macrophages, smooth muscle cells (SMCs), and extracellular matrix (ECM). The positive invariant set is found and local stability is established. Oscillatory behavior of the model solutions is also investigated. Numerical solutions show various dynamic behaviors that can occur under suitable conditions on the system parameters.
\end{abstract}

Keywords: atherosclerosis; atherosclerotic plaque growth; system stability; Hopf bifurcation

\section{Introduction}

Atherosclerosis is a serious disease occurring in the major arteries, or blood vessels, caused by a formation of fatty lesions which contain cholesterol and cell debris in the arterial wall. Lesions, which is a region that has been damaged due to injury, can form quite early in life and develop throughout one's lifetime.

Atherosclerotic plaque formation and growth in arteries are complex processes hemodynamically and mechanically. Some plaques, which are build-ups inside your arteries composed of fat, cholesterol, calcium, and other matters, remain stable throughout an individual's life, or they become unstable and can grow to such a size that they pose a health risk from stenosis, which is a partial blockage of the artery, leading to disruption. The rupture of these vulnerable plaques is thought to be responsible for most fatalities.

(c) The Author(s) 2017. This article is distributed under the terms of the Creative Commons Attribution 4.0 International License (http://creativecommons.org/licenses/by/4.0/), which permits unrestricted use, distribution, and reproduction in any medium, provided you give appropriate credit to the original author(s) and the source, provide a link to the Creative Commons license, and indicate if changes were made. 


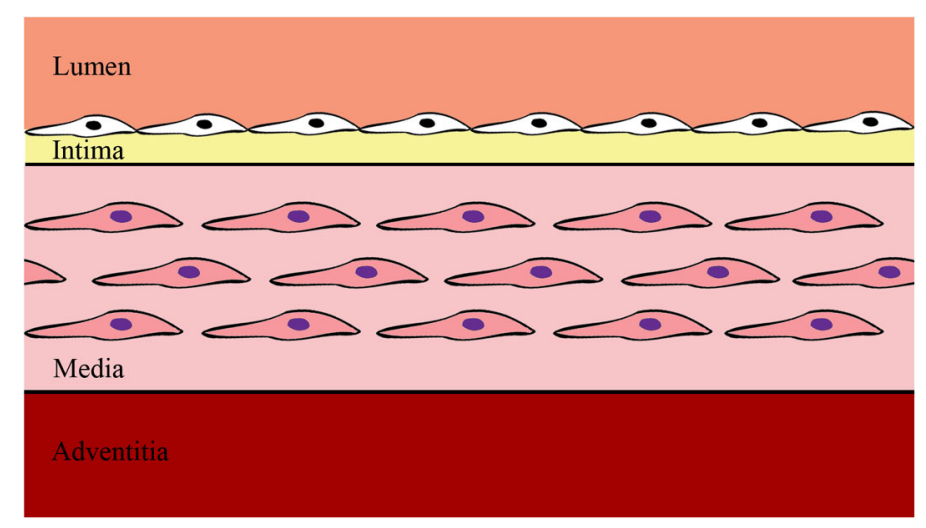

Figure 1 Diagram showing different layers of the arterial wall.

According to Davies [1], almost 73\% of deaths from myocardial infarction (heart attack) are caused by plaque rupture.

Medium-to-large arteries have three main layers. The interior boundary of the artery is lined with a layer of endothelial cells (ECs). Then there is the intima, surrounded by the media, and finally an outer layer called adventitia. Fatty streaks and plaques form in the intima. Please see Figure 1 for the diagram of these layers of the arterial wall.

According to recent statistics [2], atherosclerosis overwhelmingly causes more morbidity and mortality in the western world than other diseases. The prevention and treatment of atherosclerosis is one of the most important problems in medicine. For this reason, better understanding of the atherosclerotic plaque development has been a subject of intense investigation.

The disease process begins when LDL ('bad' cholesterol) in the bloodstream internalizes through the endothelial cells and enters the intima in the artery wall. The LDL particles then are modified by free receptors and can get oxidized into a modified form such as $o x$ idized LDL (oxLDL). The immune response following the diffusion and oxidation of LDL cholesterol urges the endothelial cells near the inflammatory area to recruit monocytes from the bloodstream, which subsequently enter the intima [3]. Once in the intima, the monocytes differentiate (or become specialized) to turn into macrophages in the artery wall and ingest the oxLDLs through the scavenger receptors on their surfaces [4].

These macrophages eventually transform into foam cells, the hallmark of the artery lesion of fatty streak. This leads to trapping of cholesterol within the artery wall. The maturation of fatty streaks into more advanced plaques produces lesions that are usually covered with a fibrous cap composed of smooth muscle cells (SMCs) and extracellular matrix (ECM) components, such as elastin and collagen [5].

The migration of SMCs responds to a chemical signal produced during the accumulation of oxLDL, foam cells, and debris. This results in the formation of a fibrous cap, a layer of connective tissue that forms an atherosclerotic plaque, which shields the lesion from the lumen. The fibrous cap encloses a lipid-rich necrotic core composed of oxLDLs, cholesterol and apoptotic or necrotic cells that are unable to obtain sufficient nutrients for survival [6]. As the inflammatory process progresses, apoptosis (cell death that occurs normally) and matrix degradation by matrix metalloproteinases (MMPs) become apparent. As inflammation escalates, accompanied by persistent foam cell recruitment and the 
ever more necrotic environment within the atherosclerotic plaque, further development and maturation of the atherosclerotic lesion ensue. This assists in the enlargement of the lipid rich core and thickening of the fibrous cap [6].

Atherosclerotic plaques can be classified into two types: stable and unstable plaques. Stable atherosclerotic plaques are characterized by a thicker layer of fibrous cap, which protects the plaque from rupture. Unstable atherosclerotic plaques are characterized by a lipid core covered by a relatively thin fibrous cap containing less extracellular matrix and vascular smooth muscle cells, often with inflammatory cells and secretion of proteinases. This may lead to rupture or fissure of the surface of the plaque, thus exposing the lipid core to the bloodstream causing thrombosis (local clotting of the blood) and many cardiovascular diseases [7].

Most modeling of plaque development involves spatial-temporal constructs because there are also fluid stresses that come into play $[3,8,9]$. Because of the effort spent in dealing with the partial differential equations, the chemistry gets reduced drastically. Our purpose is to develop the basic dynamic model that describes the interaction among vital components in the temporal dynamics of the plaque formation process to which the spatial aspect could be readily incorporated later. First, the positive invariant set is found and local stability is established. Then, Hopf bifurcation analysis is carried out to illustrate the existence of sustained oscillatory behavior of the model solutions. Next, numerical simulations are carried out to verify our theoretical predictions concerning various dynamic behaviors that can occur under suitable conditions on the system parameters. Finally, clinical interpretation and conclusion are given.

\section{System model}

Atherosclerotic plaques start with some 'insult' to the intimal layer of the cardiac artery that initiates an inflammatory response. LDLs and immune cells (mainly monocytes and T-cells) migrate from the lumen into the intima. The monocytes quickly mature into macrophages, and through a rather complicated process the LDLs are oxidized, mainly due to the pressure of the free radicals. The macrophages are now able to ingest the oxidized LDLs and become fat-ladened foam cells.

We assume there are chemotactic mechanisms, like a macrophage colony stimulating factor, that facilitate the migration of smooth muscle cells into the intima to augment the native smooth muscle cell population. A late process is the formation of the cap separating the plaques from the lumen.

The 'health' of the cap is our main concern because it is what determines stable from unstable plaque.

Our model follows the following variables: LDL concentration $\left(L_{l}\right)$, oxidized LDL concentration $\left(L_{O}\right)$, macrophages density $(M)$, smooth muscle cell density $(S)$, and two chemoattractants, one an oxidized-LDL-derived chemoattractant $\left(C_{O}\right)$, and the other a macrophage-derived chemoattractant $\left(C_{M}\right)$. We also have equations for the foam cell density $(F)$ and extracellular matrix concentration $(E)$. Figure 2 shows a schematic diagram of the process being modeled below.

The equation that models the evolution of LDL concentration is as follows:

$$
\frac{d L_{l}}{d t}=\sigma-d_{1} R_{f} L_{l}
$$




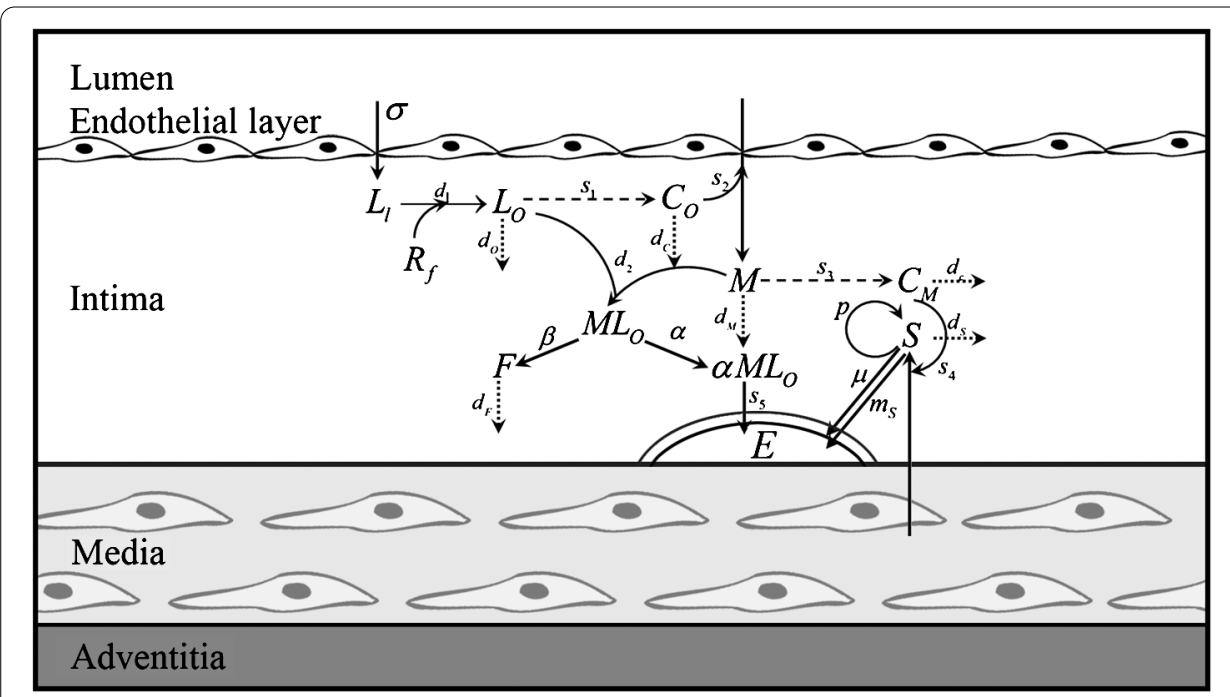

Figure 2 Diagram showing the reactions among the variables modeled in (1)-(8).

which assumes that $L_{l}$ is supplied at a constant rate, $\sigma$, by the blood and is lost through the oxidization process modeled by the last term. Here, $R_{f}$ denotes the free radicals in the oxidization according to $L_{l}+R_{f} \rightarrow \vec{d}_{1} L_{O}, d_{1}$ being the oxidation rate, and is left as a fixed parameter of the model.

The equation

$$
\frac{d L_{O}}{d t}=d_{1} R_{f} L_{l}-d_{2} M L_{O}-d_{O} L_{O}
$$

models the evolution of oxidized LDL concentration, whose source is $L_{l}$ and is lost through ingestion by macrophages with rate constant $d_{2}$. $d_{O}$ is the degradation rate of $L_{O}$. It is assumed that the uptake of $L_{O}$ is sufficiently efficient that there is no loss of $L_{O}$ to processes other than degradation.

The evolution of macrophage concentration within the compartment is modeled by

$$
\frac{d M}{d t}=s_{2} C_{O}-d_{2} M L_{O}-d_{M} M,
$$

where $s_{2}$ is the immigration rate of $M$ fostered by $C_{O}$, and $d_{M}$ is the death rate, and a percentage of oxidized LDL-ingested macrophages is lost to foam cell development ( $\beta \in$ $(0,1))$, so that the evolution of foam cells follows the following equation:

$$
\frac{d F}{d t}=\beta d_{2} M L_{O}-d_{F} F
$$

where $d_{F}$ is the death rate of foam cells and $\beta$ is the fraction of $M L_{O}$ that goes into the production of foam cells. $C_{O}$ is modeled by

$$
\frac{d C_{O}}{d t}=s_{1} L_{O}-d_{C} C_{O}
$$

in which we assume that an oxidized LDL-derived chemoattractant fosters the migration of macrophages into the compartment at the rate given by the first term in (5) with the 
Table 1 Meanings and units of variables in the model system (1)-(8)

\begin{tabular}{llr}
\hline Variable & Meaning & Unit \\
\hline$L_{1}$ & Low density lipoprotein concentration & $\mathrm{g} \mathrm{cm}^{-3}$ \\
$L_{O}$ & Oxidized low density lipoprotein concentration & $\mathrm{g} \mathrm{cm}$ \\
$M$ & Macrophage concentration & $\mathrm{g} \mathrm{cm}$ \\
$F$ & Foam cells density & $\mathrm{g} \mathrm{cm}$ \\
$C_{O}$ & Concentration of oxidized-LDL-derived chemoattractant & $\mathrm{g} \mathrm{cm}$ \\
$C_{M}$ & Concentration of macrophage-derived chemoattractant & $\mathrm{g} \mathrm{cm}$ \\
$S$ & Smooth muscle cell density & $\mathrm{g} \mathrm{cm}$ \\
$E$ & Extracellular matrix concentration & $\mathrm{g} \mathrm{cm}$ \\
\hline
\end{tabular}

rate constant $s_{1}, s_{2} C_{O}$ being the 'immigration fostering' term in the $M$-equation. $C_{O}$ can degrade at the rate $d_{C}$.

The equation for the macrophage-derived chemoattractant is

$$
\frac{d C_{M}}{d t}=s_{3} M-d_{c} C_{M}-\frac{\rho C_{M} S}{1+k_{c} C_{M}}
$$

in which $C_{M}$ is produced at the rate $s_{3}$ and naturally degrades at the rate $d_{c}$. The last term represents $C_{M}$ being captured by the smooth muscle cells $S$; that is $C_{M}$ is responsible for the movement of smooth muscle cells into the intima from the media layer with the saturation constant $k_{c}$ and the maximal rate constant $\frac{\rho}{k_{c}}$.

The smooth muscle cells follow the equation

$$
\frac{d S}{d t}=\frac{s_{4} C_{M}}{1+k_{c} C_{M}}+p S-m_{S} S-d_{S} S
$$

where the first term on the right-hand side is a 'chemotactic' immigration term with the maximal production rate $\frac{s_{4}}{k_{c}}$. The second term is a linearized proliferation term with $p$ being intrinsic growth rate, since there is a 'native' population of smooth muscle cells. The last term is an intrinsic death term with the death rate constant $d_{S} . m_{S}$ is the rate at which $S$ is lost to produce $E$.

Finally, the growth and degradation of the cap depend on the concentrations of $S$ and $M$. Extracellular matrix material (collagen) mainly comes from smooth muscle cells and provides the building material for the cap. We assume that the production rate of extracellular matrix is proportional to the concentration of smooth muscle cells, and the level of macrophages in the cap is proportional to the concentration of matrix metalloproteinases (MMPs). Hence, the portion of smooth muscle cells devoted to the cap is $m_{S} S$, and the portion of the oxidized LDL consumed macrophages affecting the cap is proportional to $d_{2} M L_{O}$; that is, $\alpha d_{2} M L_{O}$. Here, $\alpha$ is the fraction of $M L_{O}$ that goes into the production of $E$, which $\beta$ is the fraction that goes into the production of $F$ so that $\alpha+\beta=1$. Thus, the rate of growth of cap material is given by

$$
\frac{d E}{d t}=\alpha d_{2} s_{5} M L_{O} E+\left(m_{S}+\mu\right) S-d_{E} E .
$$

The long term behavior of $E$ determines whether the cap becomes vulnerable to rupturing or not. The meanings and units of the variables in our model are given in Table 1.

In the next section, we show that the model consisting of equations (1)-(8) admits positive and bounded solutions under suitable conditions on the system parameters. 


\section{Stability analysis}

First, we need to show the following lemma which ensures that, under suitable conditions, all the solutions of system (1)-(8) are nonnegative. We also identify a set $\mathcal{B}$ in $\mathbb{R}_{+}^{8}$ such that all solutions starting from $\mathcal{B}$ remain bounded.

Lemma 1 Let all parameters in (1)-(8) be positive and

$$
m_{S}+d_{S}>p
$$

and $\mathcal{B}$ be the region in $\mathbb{R}_{+}^{8}$ defined by

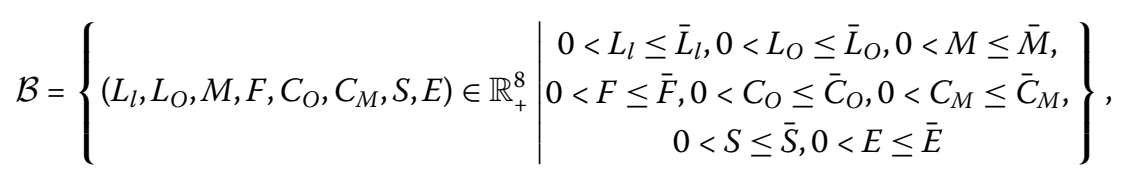

where

$$
\begin{aligned}
& \bar{L}_{l}=\frac{\sigma}{d_{1} R_{f}}, \quad \bar{L}_{O}=\frac{\sigma}{d_{O}}, \quad \bar{M}=\frac{\sigma s_{1} s_{2}}{d_{C} d_{M} d_{O}}, \quad \bar{F}=\frac{\beta d_{2} \sigma^{2} s_{1} s_{2}}{d_{F} d_{C} d_{M} d_{O}^{2}}, \quad \bar{C}_{O}=\frac{\sigma s_{1}}{d_{C} d_{O}}, \\
& \bar{C}_{M}=\frac{\sigma s_{1} s_{2} s_{3}}{d_{c} d_{C} d_{M} d_{O}}, \quad \bar{S}=\frac{\sigma s_{1} s_{2} s_{3} s_{4}}{\left(m_{S}+d_{S}-p\right) d_{c} d_{C} d_{M} d_{O}}, \\
& \bar{E}=\frac{\sigma s_{1} s_{2} s_{3} s_{4}\left(m_{S}+\mu\right)}{\left(m_{S}+d_{S}-p\right) d_{c} d_{C} d_{E} d_{M} d_{O}} .
\end{aligned}
$$

Then $\mathcal{B}$ is positive invariant and all solutions starting in $\mathcal{B}$ are uniformly bounded.

Proof First, we show that a solution starting from within $\mathcal{B}$ is positive. Let

$$
\left(L_{l}(0), L_{O}(0), M(0), F(0), C_{O}(0), C_{M}(0), S(0), E(0)\right) \in \mathcal{B} \text {. }
$$

If $L_{l}(t)$ were to become nonpositive, then there would exist $t_{0}>0$ such that $L_{l}\left(t_{0}\right)=0$ and $L_{l}(t)>0$ for any $t, 0 \leq t<t_{0}$. Then, necessarily, $\left.\frac{d L_{l}}{d t}\right|_{t=t_{0}} \leq 0$, which is a contradiction because

$$
\left.\frac{d L_{l}}{d t}\right|_{t=t_{0}}=\sigma-d_{1} R_{f} L_{l}\left(t_{0}\right)=\sigma>0
$$

Hence, $L_{l}(t)$ remains positive for all $t \geq 0$.

Next, $L_{O}(t)$ would become nonpositive if there existed $t_{0}>0$ such that $L_{O}\left(t_{0}\right)=0$ and $L_{O}(t)>0$ for any $t, 0 \leq t<t_{0}$. Then, necessarily, $\left.\frac{d L_{O}}{d t}\right|_{t=t_{0}} \leq 0$, which is a contradiction because

$$
\left.\frac{d L_{O}}{d t}\right|_{t=t_{0}}=d_{1} R_{f} L_{l}\left(t_{0}\right)-d_{2} M\left(t_{0}\right) L_{O}\left(t_{0}\right)-d_{O} L_{O}\left(t_{0}\right)=d_{1} R_{f} L_{l}\left(t_{0}\right)>0
$$

Hence, $L_{O}(t)$ remains positive for all $t \geq 0$.

If $C_{O}(t)$ were to become nonpositive, then there would exist $t_{0}>0$ such that $C_{O}\left(t_{0}\right)=0$ and $C_{O}(t)>0$ for any $t, 0 \leq t<t_{0}$. Then, necessarily, $\left.\frac{d C_{O}}{d t}\right|_{t=t_{0}} \leq 0$, which is a contradiction 
because

$$
\left.\frac{d C_{O}}{d t}\right|_{t=t_{0}}=s_{1} L_{O}\left(t_{0}\right)-d_{C} C_{O}\left(t_{0}\right)=s_{1} L_{O}\left(t_{0}\right)>0 .
$$

Hence, $C_{O}(t)$ remains positive for all $t \geq 0$.

If $M(t)$ were to become nonpositive, then there would exist $t_{0}>0$ such that $M\left(t_{0}\right)=0$ and $M(t)>0$ for any $t, 0 \leq t<t_{0}$. Then, necessarily, $\left.\frac{d M}{d t}\right|_{t=t_{0}} \leq 0$, which is a contradiction because

$$
\left.\frac{d M}{d t}\right|_{t=t_{0}}=s_{2} C_{O}\left(t_{0}\right)-d_{2} M\left(t_{0}\right) L_{O}\left(t_{0}\right)-d_{M} M\left(t_{0}\right)=s_{2} C_{O}\left(t_{0}\right)>0 .
$$

Hence, $M(t)$ remains positive for all $t \geq 0$.

Next, $F(t)$ would become nonpositive if there existed $t_{0}>0$ such that $F\left(t_{0}\right)=0$ and $F(t)>$ 0 for any $t, 0 \leq t<t_{0}$. Then, necessarily, $\left.\frac{d F}{d t}\right|_{t=t_{0}} \leq 0$, which is a contradiction because

$$
\left.\frac{d F}{d t}\right|_{t=t_{0}}=\beta d_{2} M\left(t_{0}\right) L_{O}\left(t_{0}\right)-d_{F} F\left(t_{0}\right)=\beta d_{2} M\left(t_{0}\right) L_{O}\left(t_{0}\right)>0
$$

Hence, $F(t)$ remains positive for all $t \geq 0$.

$C_{M}(t)$ would become nonpositive if there existed $t_{0}>0$ such that $C_{M}\left(t_{0}\right)=0$ and $C_{M}(t)>$ 0 for any $t, 0 \leq t<t_{0}$. Then, necessarily, $\left.\frac{d C_{M}}{d t}\right|_{t=t_{0}} \leq 0$, which is a contradiction because

$$
\left.\frac{d C_{M}}{d t}\right|_{t=t_{0}}=s_{3} M\left(t_{0}\right)-d_{c} C_{M}\left(t_{0}\right)-\frac{\rho C_{M}\left(t_{0}\right) S\left(t_{0}\right)}{1+k_{c} C_{M}\left(t_{0}\right)}=s_{3} M\left(t_{0}\right)>0 .
$$

Hence, $C_{M}(t)$ never vanishes and is positive for all $t \geq 0$.

$S(t)$ would become nonpositive if there existed $t_{0}>0$ such that $S\left(t_{0}\right)=0$ and $S(t)>0$ for any $t, 0 \leq t<t_{0}$. Then, necessarily, $\left.\frac{d S}{d t}\right|_{t=t_{0}} \leq 0$, which is a contradiction because

$$
\left.\frac{d S}{d t}\right|_{t=t_{0}}=\frac{s_{4} C_{M}\left(t_{0}\right)}{1+k_{c} C_{M}\left(t_{0}\right)}-\left(m_{S}+d_{S}-p\right) S\left(t_{0}\right)=\frac{s_{4} C_{M}\left(t_{0}\right)}{1+k_{c} C_{M}\left(t_{0}\right)}>0 .
$$

Hence, $S(t)$ never vanishes and is positive for all $t \geq 0$.

Finally, $E(t)$ would become nonpositive if there existed $t_{0}>0$ such that $E\left(t_{0}\right)=0$ and $E(t)>0$ for any $t, 0 \leq t<t_{0}$. Then, necessarily, $\left.\frac{d E}{d t}\right|_{t=t_{0}} \leq 0$, which is a contradiction because

$$
\left.\frac{d E}{d t}\right|_{t=t_{0}}=\left(m_{S}+\mu\right) S\left(t_{0}\right)+\alpha d_{2} s_{5} M\left(t_{0}\right) L_{O}\left(t_{0}\right) E\left(t_{0}\right)-d_{E} E\left(t_{0}\right)=\left(m_{S}+\mu\right) S\left(t_{0}\right)>0
$$

Hence, $E(t)$ never vanishes and is positive for all $t \geq 0$.

We next consider the model system (1)-(8) with $\left(L_{l 0}, L_{O 0}, M_{0}, F_{0}, C_{O 0}, C_{M 0}, S_{0}, E_{0}\right) \in \mathcal{B}$. From (1), we obtain

$$
L_{l}(t)=L_{l}(0) e^{-d_{1} R_{f} t}+\frac{\sigma}{d_{1} R_{f}}\left(1-e^{-d_{1} R_{f} t}\right) \leq \bar{L}_{l} e^{-d_{1} R_{f} t}+\bar{L}_{l}\left(1-e^{-d_{1} R_{f} t}\right) .
$$

Hence,

$$
L_{l}(t) \leq \bar{L}_{l}=\frac{\sigma}{d_{1} R_{f}}
$$


From (2), we can write

$$
\frac{d L_{O}}{d t} \leq d_{1} R_{f} \bar{L}_{l}-d_{O} L_{O}=\sigma-d_{O} L_{O}
$$

Then

$$
L_{O}(t) \leq L_{O}(0) e^{-d_{O} t}+\frac{\sigma}{d_{O}}\left(1-e^{-d_{O} t}\right) \leq \bar{L}_{O} e^{-d_{O} t}+\bar{L}_{O}\left(1-e^{-d_{O} t}\right)
$$

Hence,

$$
L_{O}(t) \leq \bar{L}_{O}=\frac{\sigma}{d_{O}}
$$

From (5), we can write

$$
\frac{d C_{O}}{d t} \leq s_{1} \bar{L}_{O}-d_{C} C_{O}=\frac{\sigma s_{1}}{d_{O}}-d_{C} C_{O}
$$

Then

$$
C_{O}(t) \leq C_{O}(0) e^{-d_{C} t}+\frac{\sigma s_{1}}{d_{C} d_{O}}\left(1-e^{-d_{C} t}\right) \leq \bar{C}_{O} e^{-d_{C} t}+\bar{C}_{O}\left(1-e^{-d_{C} t}\right)
$$

Hence,

$$
C_{O}(t) \leq \bar{C}_{O}=\frac{\sigma s_{1}}{d_{C} d_{O}}
$$

From (3), we can write

$$
\frac{d M}{d t} \leq s_{2} \bar{C}_{O}-d_{M} M=\frac{\sigma s_{1} s_{2}}{d_{C} d_{O}}-d_{M} M
$$

Then

$$
M(t) \leq M(0) e^{-d_{M} t}+\frac{\sigma s_{1} s_{2}}{d_{C} d_{M} d_{O}}\left(1-e^{-d_{M} t}\right) \leq \bar{M} e^{-d_{M} t}+\bar{M}\left(1-e^{-d_{M} t}\right)
$$

Hence,

$$
M(t) \leq \bar{M}=\frac{\sigma s_{1} s_{2}}{d_{C} d_{M} d_{O}}
$$

From (4), we obtain

$$
\frac{d F}{d t} \leq \beta d_{2} \bar{M} \bar{O}-d_{F} F=\frac{\beta d_{2} \sigma^{2} s_{1} s_{2}}{d_{C} d_{M} d_{O}^{2}}-d_{F} F
$$

Then

$$
F(t) \leq F(0) e^{-d_{F} t}+\frac{\beta \sigma^{2} d_{2} s_{1} s_{2}}{d_{F} d_{C} d_{M} d_{O}^{2}}\left(1-e^{-d_{F} t}\right) \leq \bar{F} e^{-d_{F} t}+\bar{F}\left(1-e^{-d_{F} t}\right)
$$


Hence,

$$
F(t) \leq \bar{F}=\frac{\beta \sigma^{2} d_{2} s_{1} s_{2}}{d_{F} d_{C} d_{M} d_{O}^{2}}
$$

Similarly, we can prove in the same fashion that

$$
\begin{aligned}
& C_{M}(t) \leq \bar{C}_{M}=\frac{\sigma s_{1} s_{2} s_{3}}{d_{c} d_{C} d_{M} d_{O}}, \\
& S(t) \leq \bar{S}=\frac{\sigma s_{1} s_{2} s_{3} s_{4}}{\left(m_{S}+d_{S}-p\right) d_{c} d_{C} d_{M} d_{O}}
\end{aligned}
$$

and

$$
E(t) \leq \bar{E}=\frac{\sigma s_{1} s_{2} s_{3} s_{4}\left(m_{S}+\mu\right)}{\left(m_{S}+d_{S}-p\right) d_{c} d_{C} d_{E} d_{M} d_{O}}
$$

Next, we let

$$
\begin{aligned}
& P=d_{2} M^{*}+d_{2} L_{O}^{*}+d_{M}+d_{O}, \\
& Q=d_{2} d_{M} M^{*}+d_{2} d_{O} L_{O}^{*}+d_{M} d_{O}, \\
& R=d_{2} s_{1} s_{2} L_{O}^{*}
\end{aligned}
$$

and $\tilde{X}^{*}=\left(L_{l}^{*}, L_{O}^{*}, M^{*}, F^{*}, C_{O}^{*}, C_{M}^{*}, S^{*}, E^{*}\right)$ be the equilibrium point of (1)-(8), where

$$
\begin{aligned}
& L_{l}^{*}=\frac{\sigma}{d_{1} R_{f}}, \quad L_{O}^{*}=\frac{\sigma}{d_{2} M^{*}+d_{O}}, \\
& M^{*}=\frac{-\left(\sigma d_{2} d_{C}+d_{C} d_{M} d_{O}\right)+\sqrt{\left(\sigma d_{2} d_{C}+d_{C} d_{M} d_{O}\right)^{2}+4 \sigma d_{n} n_{C} d_{M} s_{1} s_{2}}}{2 d_{2} d_{C} d_{M}}, \\
& C_{O}^{*}=\frac{\sigma s_{1}}{d_{C}\left(d_{2} M^{*}+d_{O}\right)}, \quad F^{*}=\frac{\beta \sigma d_{2} M^{*}}{d_{F}\left(d_{2} M^{*}+d_{O}\right)}, \quad C_{M}^{*}=\frac{\left(m_{S}+d_{S}-p\right) S^{*}}{s_{4}-k_{c}\left(m_{S}+d_{S}-p\right) S^{*}}, \\
& S^{*}=\frac{-d_{g}+\sqrt{d_{c}^{2}+4 \rho s_{3} s_{4} M^{*} /\left(m_{S}+d_{S}-p\right)}}{2 \rho}, \quad E^{*}=\frac{\left(m_{S}+\mu\right)\left(d_{2} M^{*}+d_{O}\right) S^{*}}{\alpha \sigma d_{2} s_{5} M^{*}+d_{E}\left(d_{2} M^{*}+d_{O}\right)} .
\end{aligned}
$$

By using the Routh-Hurwitz criteria, the following theorem can be shown.

Theorem 2 If (9) holds and

$$
P\left(d_{C}^{2}+d_{C} P+Q\right)>R
$$

then $\tilde{X}^{*}$ is locally asymptotically stable.

Proof By finding the determinant of the Jacobian matrix of the model system (1)-(8) at $\tilde{X}$, the characteristic equation associated with system (1)-(8) can be written as

$$
\left(\lambda+d_{1} R_{f}\right)\left(\lambda+d_{F}\right)\left(\lambda+d_{E}+\alpha d_{2} s_{5} M^{*} L_{O}^{*}\right)\left(\lambda^{2}+a_{1} \lambda+a_{2}\right)\left(\lambda^{3}+b_{1} \lambda^{2}+b_{2} \lambda+b_{3}\right)=0,
$$


where

$$
\begin{aligned}
& a_{1}=\left(d_{c}+\frac{\rho S^{*}}{\left(1+k_{c} C_{M}^{*}\right)^{2}}\right)+\left(m_{S}+d_{S}-p\right), \\
& a_{2}=\left(d_{g}+\frac{\rho S^{*}}{\left(1+k_{c} C_{M}^{*}\right)^{2}}\right)\left(m_{S}+d_{S}-p\right)+\frac{\rho s_{4} C_{M}^{*}}{\left(1+k_{c} C_{M}^{*}\right)^{3}}, \\
& b_{1}=d_{C}+P, \\
& b_{2}=d_{C} P+Q \\
& b_{3}=d_{C} Q+R .
\end{aligned}
$$

We first find directly that the three eigenvalues of (1)-(8) are clearly negative. In fact, from the first three factors in (11), we have

$$
\begin{aligned}
& \lambda_{1}=-d_{1} R_{f}<0, \\
& \lambda_{2}=-d_{F}<0
\end{aligned}
$$

and

$$
\lambda_{3}=-d_{E}-\alpha d_{2} s_{5} M^{*} L_{O}^{*}<0 .
$$

We then consider the factor

$$
\lambda^{2}+a_{1} \lambda+a_{2}
$$

We see that $a_{1}>0$ and $a_{2}>0$ since (9) holds. Hence, the eigenvalues $\lambda_{i}, i=4,5$, which are the roots of (14), also have negative real parts by using the Routh-Hurwitz criteria.

We next consider the polynomial

$$
\lambda^{3}+b_{1} \lambda^{2}+b_{2} \lambda+b_{3}
$$

We observe that $b_{1}=d_{C}+P>0, b_{2}=d_{C} P+Q>0$ and $b_{3}=d_{C} Q+R$ and since (10) holds, we have

$$
\begin{aligned}
b_{1} b_{2}-b_{3} & =\left(d_{C}+P\right)\left(d_{C} P+Q\right)-\left(d_{C} Q+R\right) \\
& =P\left(d_{C}^{2}+d_{C} P+Q\right)-R>0 .
\end{aligned}
$$

Hence, the eigenvalues for $\lambda_{i}, i=6,7,8$, which are the roots of (15), also have negative real parts, by the Routh-Hurwitz criteria.

Therefore, the equilibrium point $\tilde{X}^{*}$ is locally asymptotically stable as claimed.

\section{Sustained oscillation}

We next show that the model system (1)-(8) admits periodic solutions through a Hopf bifurcation of the equilibrium point $\tilde{X}^{*}=\left(L_{l}^{*}, L_{O}^{*}, M^{*}, F^{*}, C_{O}^{*}, C_{M}^{*}, S^{*}, E^{*}\right)$. 
Theorem 3 Using the symbols defined in (12) and (13) in Theorem 2, if (9) holds and

$$
P\left(d_{C}^{2}+d_{C} P+Q\right)<R
$$

then system (1)-(8) undergoes a Hopf bifurcation. A limit cycle of system (1)-(8) will emerge and can be observed in the vicinity of the equilibrium point $\tilde{X}^{*}$.

Proof Following from the proof of Theorem 2, the roots $\lambda_{i}, i=1,2, \ldots, 5$, of the characteristic equation (11), that are the roots of the first five factors of (11), will have negative real parts since (9) holds. Now, for the factor in (15), the conditions

$$
\begin{aligned}
& b_{1}=d_{C}+P>0, \\
& b_{2}=d_{C} P+Q>0
\end{aligned}
$$

and

$$
b_{3}=d_{C} Q+R>0
$$

still hold.

If $\lambda_{6}$ is any root of the cubic (15), then we may factor out $\left(\lambda-\lambda_{6}\right)$ using polynomial long division to obtain

$$
\lambda^{3}+b_{1} \lambda^{2}+b_{2} \lambda+b_{3}=\left(\lambda-\lambda_{6}\right)\left(\lambda^{2}+\left(b_{1}+\lambda_{6}\right) \lambda+\left(b_{2}+b_{1} \lambda_{6}+\lambda_{6}^{2}\right)\right) .
$$

The quadratic factor in (17) has the roots

$$
\lambda_{7,8}=\frac{-b_{1}-\lambda_{6} \pm \sqrt{b_{1}^{2}-4 b_{2}-2 b_{1} \lambda_{6}-3 \lambda_{6}^{2}}}{2} .
$$

When $b_{1}=-\lambda_{6}$, then

$$
\lambda_{7,8}= \pm i \sqrt{b_{2}}
$$

which is pure imaginary. Moreover, since $\lambda_{6}$ is a root of (15), we have

$$
\lambda_{6}^{3}+b_{1} \lambda_{6}^{2}+b_{2} \lambda_{6}+b_{3}=0
$$

from which we can write

$$
\lambda_{6}\left(\lambda_{6}^{2}+b_{1} \lambda_{6}+b_{2}\right)=-b_{3}
$$

and hence

$$
\lambda_{6}=-\frac{b_{3}}{b_{2}+b_{1} \lambda_{6}+\lambda_{6}^{2}}<-\frac{b_{3}}{b_{2}}
$$




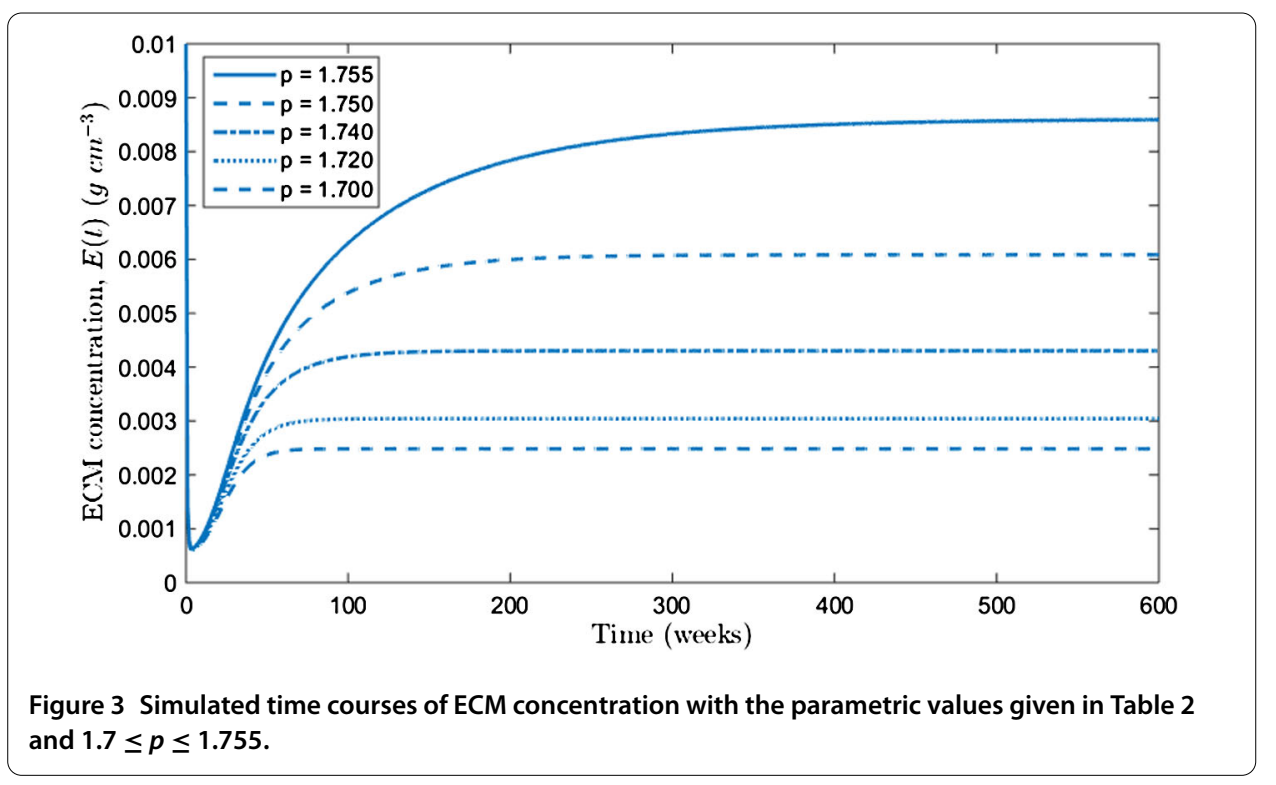

when $-b_{1}-\lambda_{6}>0$. From the definitions of $b_{1}, b_{2}$, and $b_{3}$, we have

$$
\begin{aligned}
b_{1} b_{2} & =\left(d_{C}+P\right)\left(d_{C} P+Q\right) \\
& =P\left(d_{C}^{2}+d_{C} P+Q\right)+d_{C} Q \\
& <R+d_{C} Q=b_{3}
\end{aligned}
$$

since (16) holds. Hence

$$
-\frac{b_{3}}{b_{2}}>-b_{1}
$$

Thus, a Hopf bifurcation occurs [8].

\section{Model simulation}

In the following, we show simulated solutions of the system model (1)-(8), in which some of the parameter values have been chosen based on the work of Hao and Friedman [10]. Some parameters have been chosen, however, so that the necessary conditions for specific dynamic behavior are satisfied. We simulated equations (1)-(8) using parametric values that satisfy the conditions in Theorem 2, obtaining a solution trajectory which tends toward a stable equilibrium point as seen in Figure 3, where ECM concentration is plotted against time for different values of intrinsic growth rate $p$ of the smooth muscle cells. The parametric values used here are given in Table 2. The simulated time courses of ECM, $E(t)$, are compared for $1.7 \leq p \leq 1.755$ in which case the equilibrium point is stable. When $p$ is small, the level of ECnrops to a very low level. This is the situation where the fibrous cap becomes very thin. When the caps are too thin, they are vulnerable plaques and have a higher percentage of macrophages than SMCs. Conversely, thick plaques with lower percentage of macrophages are more stable.

Physically, if there is a time $\tau>0$ such that for some predetermined $\delta>0$ ECM level decreases to the point that $E(T) \leq \delta$, the plaque would be expected to reach a point of 
Table 2 Parametric values used in the model simulations shown in Figures 3-5

\begin{tabular}{|c|c|c|c|c|}
\hline Parameter & Meaning & Value (Figures 3, 4) & Value (Figure 5) & Unit \\
\hline$\overline{d_{1}}$ & Reaction rate constant in (1) & $1.520 \times 10^{4}$ & $1.520 \times 10^{3}$ & $\mathrm{~g}^{-1} \mathrm{~cm}^{3} \mathrm{wk}^{-1}$ \\
\hline$d_{2}$ & Variation constant in (2) & 1.429 & 0.8024 & $\mathrm{~g}^{-1} \mathrm{~cm}^{3} \mathrm{wk}^{-1}$ \\
\hline$d_{0}$ & Degradation rate of $L_{O}$ & $6.048 \times 10^{-1}$ & 0.01 & $\mathrm{wk}^{-1}$ \\
\hline$d_{M}$ & Death rate of $M$ & $6.048 \times 10^{-1}$ & 0.01 & $w^{-1}$ \\
\hline$d_{F}$ & Death rate of $F$ & $4.285 \times 10^{-3}$ & 0.25 & $w^{-1}$ \\
\hline$d_{C}$ & Degradation rate of $C_{O}$ & 0.01 & 0.01 & $w^{-1}$ \\
\hline$d_{c}$ & Degradation rate of $C_{M}$ & 0.01 & 0.1 & $w k^{-1}$ \\
\hline$d_{s}$ & Intrinsic death rate of $S$ & 1.08 & 0.1 & $w k^{-1}$ \\
\hline$d_{E}$ & Removal rate of $E$ & 0.5 & 0.05 & $w^{-1}$ \\
\hline$k_{c}$ & Saturation constant of migration & $10^{-4}$ & $10^{-2}$ & $w^{-1}$ \\
\hline$m_{s}$ & Removal rate of $S$ to form $E$ & 1.68 & 0.5 & $w^{-1}$ \\
\hline$s_{1}$ & Production rate of $C_{O}$ & $6.05 \times 10^{-3}$ & $1.520 \times 10^{3}$ & $w^{-1}$ \\
\hline$s_{2}$ & Immigration rate of $M$ fostered by $C_{O}$ & 10.2 & 0.8024 & $w^{-1}$ \\
\hline$s_{3}$ & Production rate of $C_{M}$ & 0.1814 & 0.01 & $w^{-1}$ \\
\hline$S_{4}$ & $\begin{array}{l}\text { Proportionality constant in production rate } \\
\text { of } S \text { due to migration }\end{array}$ & 1.814 & 0.01 & $w^{-1}$ \\
\hline$S_{5}$ & Variation constant in production rate of $E$ & 6.0 & 0.25 & $w^{-1}$ \\
\hline$R_{f}$ & Number of free radicals in oxidation process & 0.02772 & 0.01 & $\mathrm{mg} / \mathrm{ml}$ \\
\hline$\alpha$ & Fraction of $M L_{O}$ that goes into $E$ production & 0.5 & 0.6 & - \\
\hline$\beta$ & Fraction of $M L_{O}$ that goes into $F$ production & 0.5 & 0.4 & - \\
\hline$\mu$ & Proportionality constant in (8) & 6.05 & 0.00238 & $w k^{-1}$ \\
\hline$\rho$ & Migration rate of $S$ at vanishing $C_{M}$ & 7.5 & 0.001 & $\mathrm{~g}^{-1} \mathrm{~cm}^{-3} \mathrm{wk}^{-1}$ \\
\hline$\sigma$ & Growth rate of $L_{1}$ & $2.016 \times 10^{3}$ & $2.016 \times 10^{3}$ & $\mathrm{~g}^{-1} \mathrm{~cm}^{-3} \mathrm{wk}^{-1}$ \\
\hline$p$ & Proliferation rate of $S$ & {$[1.700,1.765]$} & 0.4 & $w k^{-1}$ \\
\hline
\end{tabular}

'rupture'. The rupture of these vulnerable plaques is discovered to be responsible for most human fatalities suffering from heart diseases. According to Davies (1992) [1], almost 73\% of deaths from myocardial infarction (heart attack) are caused by plaque rupture.

On the other hand, some plaques may remain stable throughout a person's life, or the plague may become of such a size that it poses a serious risk to a patient's health due to stenosis, a partial blockage of the artery which causes blood flow disruption. Such a situation is simulated by our model shown in Figure 4, where the conditions that ensure system stability stipulated in Theorem 2 are violated. ECM level thus continues to grow substantially increasing the risk of serious health complications for that person.

Figure 5 shows the simulated time course of our model system (1)-(8) in the case that conditions in Theorem 3 are satisfied and a Hopf bifurcation occurs. The parametric values used here are given in Table 2. EMC level is seen to oscillate periodically as time progresses as theoretically predicted. Periodicity corresponds to biological fluctuations or sustained oscillations that are found in most physiological systems. They are the consequence of a medley of the fluctuating environment. A model that does not admit periodic solutions, for this reason, is not an adequate representation of the system of interest. Our model has been shown to exhibit sustained oscillation and thus serves a suitable representation of the plaque formation process.

\section{Conclusion}

Atherosclerosis is an extremely dangerous disease due to the fact that the process of narrowing and hardening of the arteries occurs slowly and can take several decades before it shows any symptoms. Apart from leading to heart attacks and strokes, which cause thousands of deaths annually, atherosclerosis also leads to kidney failure, blindness, and even impotence [9]. Mathematical modeling can shine some light on this stealthy disease in order to learn how to prevent its often hidden complications. 


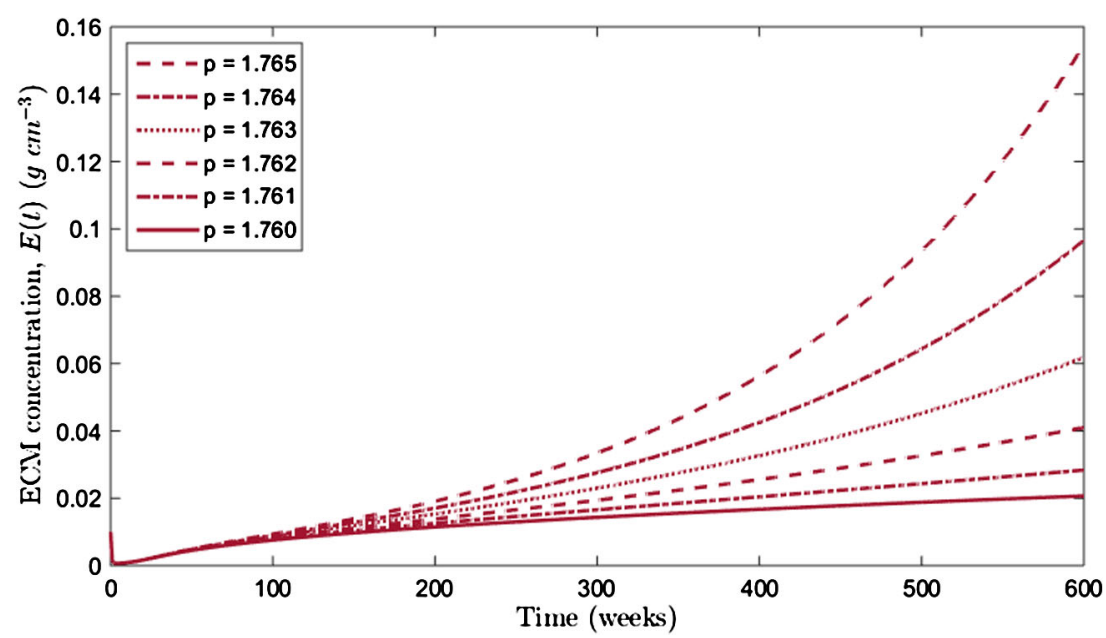

Figure 4 Simulated time courses of ECM concentration with the parametric values given in Table 2 and $p \geq 1.76$ in which case conditions in Theorem 2 are violated and the equilibrium is unstable.

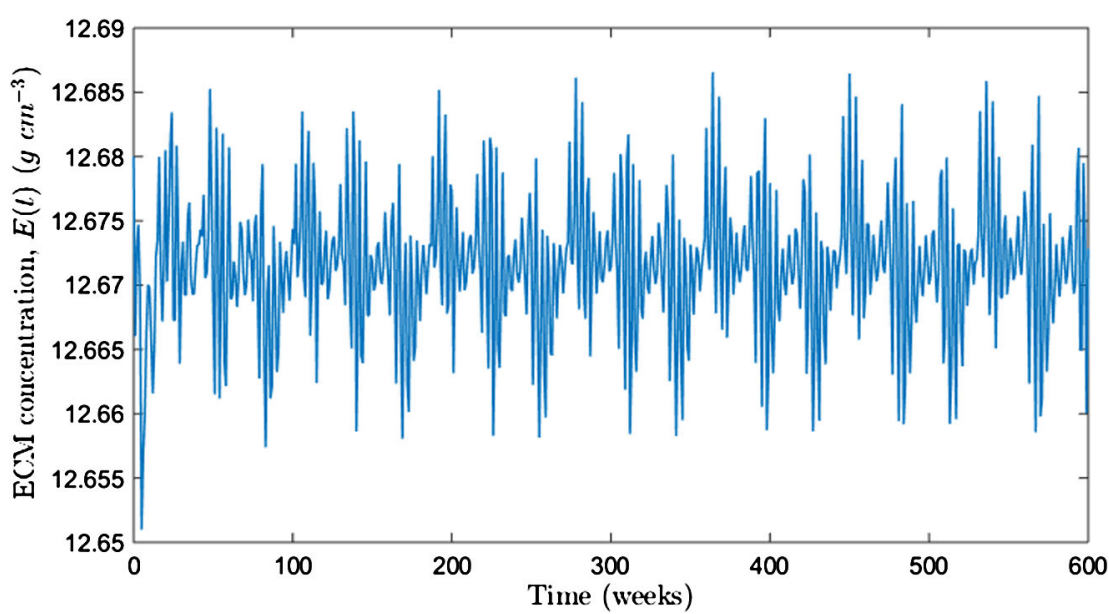

Figure 5 A simulated time course of EMC concentration when parametric values, given in Table 2, satisfy the conditions in Theorem 3 and the solution of (1)-(8) eventually oscillates periodically.

In this paper, we have constructed and analyzed a model of the process of atherosclerotic plaque formation. The system model is capable of simulating various dynamic behaviors in different cases which have been observed clinically. Ranges of physical parameters that delineate unstable from stable atherosclerotic plaques have been identified.

Including more chemistry considerations into our model, we are able to discover critical conditions that delineate different dynamic behaviors exhibited by the system. Specifically, there are two main types of atherosclerotic lesions: the stable plaque which simply impedes blood flows and an unstable one which is vulnerable to rupture. Our model identifies the proliferation rate $p$ of the smooth muscle cells as the critical physical parameter that plays the most important role in maintaining the system's stability. If $m_{S}+d_{S}>p$, the system is stable, but if $p$ increases beyond $m_{S}+d_{S}$, the system may become unstable, posing higher risk of coronary heart diseases. 
Insights gained from our investigation are expected to form a basis for further research to better understand the build-up of plaque in the arteries. Deeper comprehension of the process may lead us to a new way to treat this life threatening disease. Rather than trying to reduce the build-up by reducing LDL cholesterol levels in blood serum, regression and stabilization of plaque, not LDL cholesterol, may become the new approach in the treatment and prevention of cardiovascular disease, the leading cause of death in the U.S. and around the world [11].

\section{Acknowledgements}

Both authors are supported by a research grant from the Centre of Excellence in Mathematics, Commission on Higher Education, Thailand.

\section{Competing interests}

The authors declare that they have no competing interests.

\section{Authors' contributions}

Both authors were involved in the derivation of the model with the kind advice of Prof. Jonathan Bell, now retired from the University of Maryland Baltimore County. The first author carried out the model analysis concerning boundedness and stability of its solutions, as well as its numerical simulations. The second author carried out the bifurcation analysis and clinical interpretation of the results.

\section{Author details}

${ }^{1}$ Department of Mathematics and Statistics, Faculty of Science and Technology, Thammasat University, Pathum Thani, Thailand. ${ }^{2}$ Centre of Excellence in Mathematics, PERDO, CHE, Bangkok, Thailand. ${ }^{3}$ Department of Mathematics, Faculty of Science, Mahidol University, Rama 6 Road, Bangkok, Thailand. ${ }^{4}$ Department of Mathematics and Statistics, University of Mayland Baltimore County, Baltimore, MD, USA.

\section{Publisher's Note}

Springer Nature remains neutral with regard to jurisdictional claims in published maps and institutional affiliations.

Received: 24 January 2017 Accepted: 20 June 2017 Published online: 10 July 2017

\section{References}

1. Davies, MJ: Anatomic features in victims of sudden coronary death. Coronary artery pathology. Circulation 85(Suppl 1), I19-124 (1992)

2. Massera, D, Graf, L, Barba, S, Ostfeld, R: Angina rapidly improved with a plant-based diet and returned after resuming a western diet. J. Geriatr. Cardiol. 13(4), 364-366 (2016)

3. Bulelzai, MAK, Dubbeldam, JL: Long time evolution of atherosclerotic plaques. J. Theor. Biol. 297, 1-10 (2012)

4. Winckers, $K$, ten Cate, $H$, Hackeng, TM: The role of tissue factor pathway inhibitor in atherosclerosis and arterial thrombosis. Blood Rev. 27(3), 119-132 (2013)

5. Hundal, RS, Salh, BS, Schrader, JW, Gómez-Muñoz, A, Duronio, V, Steinbrecher, UP: Oxidized low density lipoprotein inhibits macrophage apoptosis through activation of the PI 3-kinase/PKB pathway. J. Lipid Res. 42(9), 1483-1491 (2001)

6. Fok, PW: Growth of necrotic cores in atherosclerotic plaque. Math. Med. Biol. 29(4), 301-327 (2012)

7. Finn, AV, Nakano, M, Narula, J, Kolodgie, FD, Virmani, R: Concept of vulnerable/unstable plaque. Arterioscler. Thromb. Vasc. Biol. 30(7), 1282-1292 (2010)

8. Ibragimov, A, Ritter, L, Walton, JR: Stability analysis of a reaction-diffusion system modeling atherogenesis. SIAM J. Appl. Math. 70(7), 2150-2155 (2010)

9. Calvey, V, Houot, JG, Meunier, N, Raoult, A, Rusnakova, G: Mathematical and numerical modeling of early atherosclerotic lesions. ESAIM Proc. 30, 1-14 (2010)

10. Hao, W, Friedman, A: The LDL-HDL profile determines the risk of atherosclerosis: a mathematical model. PLoS ONE 9(3), e90497 (2014)

11. Hassard, BD, Kazarinoff, ND, Wan, YH: Theory and Applications of Hopf Bifurcation, London Mathematical Society Lecture Note Series, vol. 41. Cambridge University Press, Cambridge (1981) 